advances in supernova research brought about by observations of SN 1987A.

Different readers may want different treatment of the subjects, depending on their mathematical backgrounds. Longair's discussion of Einstein's general theory of relativity, for example, provides sets of equations that may scare off non-physicists. But most of his treatments are non-mathematical, and many are humanized with quotations. For example, Longair not only points out that Jocelyn Bell first recognized the signals we now know as pulsars, but describes how he was present when the Soviet astrophysicist Iosif Shklovsky told her, ${ }^{\alpha}$ Miss Bell, you have made the greatest astronomical discovery of the twentieth century" (at least as far as 1970, when the encounter took place). Those familiar with the history of the discussion over credit for the discovery may note that he gives Antony Hewish, Bell's supervisor who shared the 1974 Nobel prize for physics for his work on pulsars, an acknowledgement for reading the chapter from which this book sprang.

Longair also tells the story of an underground seminar (literally, given its location in the basement of the Leiden Observatory in the Netherlands) in 1944, at which Jan Oort asked a young $\mathrm{H}$. C. van der Hust to calculate whether any spectral lines would be detectable in the radio part of the spectrum. Longair's discussion describes the result (a spectral line at $21 \mathrm{~cm}$ ) and explains how it was used to map the quasi-spiral arms of the Milky Way. He then describes the subsequent detection of 125 different molecules in the interstellar matter, and explains how the unexpected relationships of their abundances led researchers to deduce how the molecules were formed.

The section on exoplanets - unfortunately only a subsection of the chapter on the interstellar medium - explains the importance of high-precision spectroscopy, and starts with the pulsar planets discovered by Aleksander Wolszczan and Dale Frail. Longair then discusses the first optical detections of exoplanets by Michel Mayor and Didier Queloz, and a chapter endnote directs readers to The Extrasolar Planets Encyclopaedia if they want to keep up-to-date. I think it would have been fair to also mention the extensive work in the field by Geoff Marcy and Paul Butler, and their encydopaedic website at www.exoplanets.org.

Little of astrophysics and cosmologyescapes the gaze of Longair, a former astronomer royal for Scotland who now heads the Cavendish Laboratory in Cambridge, UK. Readers, especially those already familiar with many of the topics, will enjoy his prose. Certainly all graduate students in the field should read this book. And anyone interested in the history of science would enjoy it as bedside reading if they were willing to skip the equations.

Jay M. Pasachoff was president of the

International AstronomicalUnion's Commission onEducation and Development, and is at the Hopkins Observatory, Williams College, Williamstown, Massachusetts O1267-2565, USA.

\section{Failing the ailing}

\section{Bad Medicine: Doctors Doing Harm Since \\ Hippocrates \\ by David Wootton \\ Oxford University Press: 2006. 320 pp. £16.99, \$25}

\section{Andrew Scull}

David Wootton has made a truly remarkable discovery that he can't wait to share with the rest of us: medicine didn't work very well for millennia. Indeed, it almost certainly didn't work at all. For most of recorded history, it seems, doctors secretly did little except harm their patients while pretending to cure them. "Before 1865 all medicine was bad medicine," he writes in his book, appropriately called $\mathrm{Bad}$ Medicine. But then came Joseph Lister and antisepsis, Louis Pasteur and germ theory, and Alexander Fleming (or was it Howard Florey?) and penicillin. And from that point on, science was in place, medicine worked, and we entered an era of continuing progress and enlightenment.

Unfortunately, Wootton laments, benighted medical historians have somehow overlooked this state of affairs. Instead they have either constructed narratives of progress when medicine wasn't progressing, or (in a perverse reversal extending over three decades now) have decided that it's wrong to write tales of heroes and villains that describe an onwardsand-upwards march to scientific nirvana.

The sources of this historiographic perversity are never clearly articulated, nor are many of the offending historians identified by name, for Wootton's is a blanket condemnation. He alone has been privileged to see the true state of affairs, and henceforth the history of disease and its treatment will have to be radically recast in the light of his fundamental new insight. For the notion that there is any sort of continuity in the medical enterprise is fundamentally mistaken, he declares: we need to develop a history of error, albeit often honest error, followed by a new history of triumph. This latter alone constitutes "the modern history of medicine, grounded in constantly developing scientific understanding".

A small fraction of the book is devoted to medicine's successes: Lister's development of antiseptic surgery, John Snow's work on cholera in the mid-nineteenth century, and the advent of antibiotics, especially penicillin. For the most part, however, Wootton's eye falls on medicine's failures and missteps. For two thousand years, the galenic and hippocratic approaches dominated Western medicine. The remedies they proffered, Wootton reminds us, were painful and actively harmful, except to the degree that they produced placebo effects. Of course, there were developments in the understanding of human biology and in medical technology in these years: Andreas Vesalius produced his stunning work on human anatomy; William Harvey discovered the circulation of the blood; Antonie van Leeuwenhoek and others used microscopes to reveal the world of the infinitely small; René Laennec invented the stethoscope; and François Magendie, Claude Bernard and others used unspeakably cruel vivisections to explore the physiology of the body. But none of these developments led to therapeutic progress, and those that might have done so (such as the ability to see germs) went unexploited for decades, even centuries.

The brief sketches Wootton provides of these and other episodes in the long reign of error are rendered deftly and vividly. When he

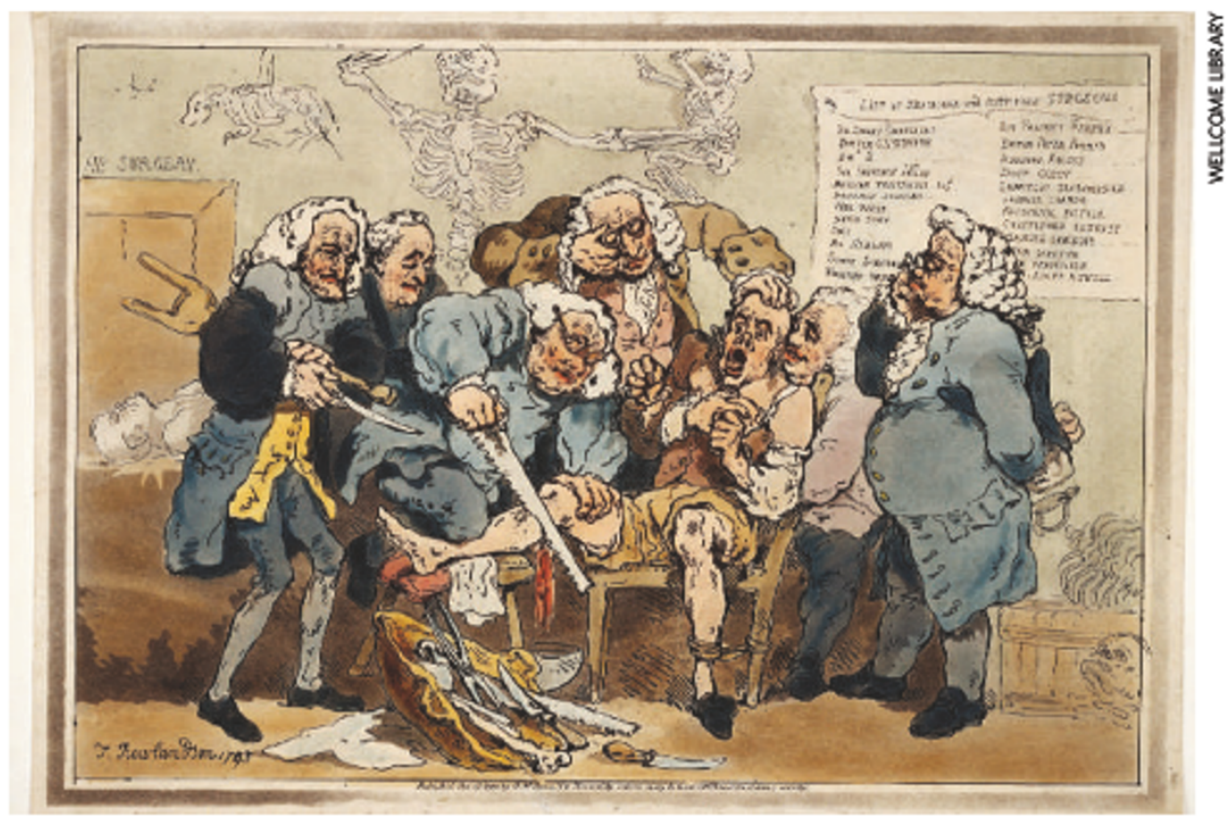

A saw point? Some medical practices may have done more harm than good. 
attempts to address larger themes, however, things do not always go so well. Frequently he points to missed opportunities to exploit the implications of technical advances and key discoveries. He cites as examples the long hiatus between the discovery of bacteria and the link to infectious disease, and the shorter but still significant gap between the first observations of the antibacterial effects of Penicillium moulds in the 1870 s and Florey's development of a therapy that worked in the 1940s.

But Wootton's attempts to blame these failures on the blinkered self-interest of medical men - “doctors were determined no scientific discovery would alter their traditional therapies of bleeding, purging, and vomiting ${ }^{\prime}-$ are crude and unsatisfying. He claims that medical historians have glorified the link between the laboratory and advances in basic science but overlooked the fact that these discoveries led to no immediate therapeutic advance. But this is simply false, as would be made clear by a quick perusal of such sources as William Bynum's Science and the Practice of Medicine in the Nineteenth Century (Cambridge University Press, 1994) or John Harley Warner's The Therapeutic
Perspective (Harvard University Press, 1986). His global characterization of the state of medical history strikes me as woefully wide of the mark - the subtle relationship between science and medicine requires a far deeper understanding than anything on offer here. And his assertion that the history of modern medicine can be reduced to a paean to scientific progress is a recipe for bad scholarship, of which there is already far too much in the world.

Andrew Scull is in the Department of Sociology,

University of California, San Diego, 9500 Gilman Drive, La Jolla, California 92093-0533, USA.

\section{A miracle in sight}

\section{Adam Elsheimer painted the starry heavens in 1609.}

\begin{abstract}
Martin Kemp
The lynx is renowned for its sharp sight. So when the Accademia dei Lincei in Rome bound its inaugural documents into a single volume in 1603 , the young German artist AdamElsheimer provided a painted image of a lynx as its frontispiece. Founded by the Roman nobleman Federico Cesi, the new academy was dedicated to the sciences of Earth and the heavens, and provided a vital forum for Galileo and a cluster of leading intellectuals who were reforming the way thatscience was conducted.

Elsheimerhad travelled a long way in a short time, both geographically and intellectually. The son of a tailor in Frankfurt, he travelled via Venice to Rome, where he settled by 1600 . He was quickly cultivated by a circle of avant-garde thinkers and artists, including the Rubens brothers, Peter Paul (the painter) and Philipp (ahumanistscholar). The former wroteemotionally on Elsheimer's early death in 1610 that .
\end{abstract} "he had no equal in small figures, in landscapes, and in many other subjects".

AllElsheimer's paintings are indeed small. They are of extraordinary visual intensity and demand unrelenting scrutiny if they are to disclose their secrets. For this reason, each visitor to the definitive exhibition of his paintings at the Edinburgh Art Festival, which closed earlier this month, was supplied with a magnifying glass. The paintings can now be seen at Dulwich Picture Gallery in London until 3 December.

The science of the Accademia dei Lincei was characterized by intense attention to visual phenomena. Within a few years of its founding, the eye was to be amplified by both the telescope and the microscope. Elsheimer's paintings declare that he was deeply immersed in this culture of taking sight as far as it would go.

Mostnotable in this respect is his

政

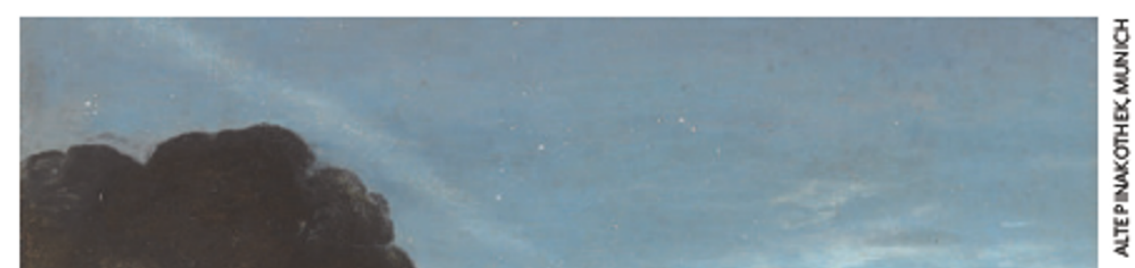

